\title{
Resource allocation based on integer programming and game theory in uplink multi-cell cooperative OFDMA systems
}

\author{
Zhao Hou*, Yueming Cai and Dan Wu
}

\begin{abstract}
In this article, we propose a semi-distributed resource allocation framework for the resource optimization in multicell uplink cooperative orthogonal frequency division multiplexing systems. Specifically, we model the resource allocation framework as an optimal problem. This optimization problem is divided into two steps. First, using integer programming, we achieve the joint relay selection and subcarrier allocation based on maximizing system sum rate in a centralized way. Second, the distributed power allocation is achieved based on game theory, for cooperative and non-cooperative users, respectively. For cooperative mobile stations, an improved utility is proposed to regulate power allocation in the two time slots. Besides the existence of Nash equilibrium (NE), a new approach for the strict mathematical proof of the uniqueness of NE is proposed. Simulation results demonstrate that the proposed algorithm successfully combines the merits of centralized and distributed framework. It can effectively make use of relays to enhance the sum rate of users as well as achieve the fairness among users.
\end{abstract}

Keywords: cooperative OFDMA systems, inter-cell interference, semi-distributed resource allocation framework, game theory, progressive optimization, Nash equilibrium

\section{Introduction}

Owing to its potential to realize high-rate and reliable communications over wireless channels, cooperative orthogonal frequency division multiplexing (OFDM) technology has drawn extensive attention in recent years as a critical 4G technology. The performance of an OFDMA system depends on careful resource management, including subcarrier allocation and power control. However, the introduction of cooperative technology, which enhances the system performance by achieving the benefits of spatial diversity, imposes more complexity on resource management. Current resource allocation algorithms in cooperative OFDMA systems can be divided into centralized and distributed categories [1-9]. The former cannot ensure fairness among users and may lead to the near-far effect, the disproportion of resource allocation caused by the channel state associated with the different locations of the users $[4,5]$. The latter is constrained with worse efficiency and more complexity, and asks for more channel overheads.

\footnotetext{
* Correspondence: konghoumingfeng@hotmail.com

* Correspondence: konghoumingfeng@hotmail.com
Institute of Communication Engineering, PLA University of Science and Technology, Nanjing, China
}

Keunyoung's [1] scheme is pursing for maximizing the OFDMA system sum rate under the power constraint. It has been accepted as the optimal solution that each subcarrier was allocated to the user with the marginal rate. Zhang et al. [2] study the relay selection, subcarrier and power allocation in cooperative OFDMA system under a QoS requirement. But, as centralized allocation schemes, these two schemes result in near-far effect because they aim at optimizing the system performance rather than the performance of each user. Therefore, subcarrier and power are allocated to users whose channel states are better, and users who have worse channel state achieve poor wireless transmission since they obtain poor wireless resources [4].

Game theory, a mathematical methodology traditionally applied in micro-economic studies, has found its applications in various aspects of communication engineering as an effective tool for studying conflictive and cooperative issues among several actors [10], including distributed resource allocation in OFDMA systems [3-9]. Han et al. [3] introduce a game-based power minimization model in a multi-cell OFDMA system. Wu et al. [4] 
and $\mathrm{Yu}$ et al. [5] focus on a subcarrier and power allocation scheme in uplink OFDMA systems, where each user not only has the different power constraint, but also is limited by different rate requirements. In [6], under a cooperative OFDMA scenario, the authors build up the interference channel model based on amplify-and-forward (AF) and decode-and-forward (DF) modes, and study power control scheme based on a two-stage game. But, the studies in $[3,6]$ do not take subcarrier allocation into account, and in [3] the authors do not introduce the relay nodes. Authors of [4-6] consider a single-cell scenario, i.e. absence of consideration of inter-cell interference. Lee and Yum [7] introduce a novel framework to find the necessary and sufficient condition for Paretoefficiency. The resource allocation algorithm developed by Lang et al. [8] aims at minimizing the users' transmitting power in a multi-cell OFDMA system based on game theory under the constraint of the peak value of power. However, this algorithm cannot ensure convergence. $\mathrm{Yu}$ et al. [9] propose a game approach for distributed power allocation in a multi-cell downlink cooperative OFDMA system, but the relay selection and subcarrier allocation are not considered. In addition, existing approaches [11] to prove the uniqueness of a game-based resource allocation scheme are of high complexity.

In this article, we propose a semi-distributed resource allocation framework for the resource optimization in multi-cell uplink cooperative OFDM systems in DF mode. Specifically, we model the resource allocation framework as an optimal problem. This optimization problem is divided into two steps. First, using integer programming, we achieve the joint relay selection and subcarrier allocation based on maximizing system sum rate in a centralized way. Second, the distributed power allocation is achieved based on game theory, for cooperative and non-cooperative users, respectively. For cooperative mobile stations, an improved utility is proposed to regulate power allocation in the two time slots. Besides the existence of Nash equilibrium (NE), a new approach for the strict mathematical proof of the uniqueness of NE is proposed. Simulation results demonstrate that the proposed algorithm successfully combines the merits of centralized and distributed framework. It can effectively make use of relays to enhance the sum rate of users as well as achieve the fairness among users.

The rest of the article is organized as follows: Section 2 describes the channel and system model. Section 3 states the framework of the progressive optimization, which is composed of the joint relay selection and subcarrier allocation, the game approach for distributed power allocation scheme, the existence and uniqueness of NE as well as the algorithm framework. Simulation results are given and analyzed in Section 4. Finally, concluding remarks in Section 5 end the article.

\section{System model}

We consider a multi-cell cooperative OFDMA scenario. In each cell, there is one base station, multiple users and multiple relays. Each user can communicate with the base station directly or through a certain relay. All of the orthogonal subcarriers are available in each cell, and each of them can exclusively be allocated to a certain user [9]. Therefore, inter-cell interference originates from co-channel users in adjacent cells.

Assume that there is a cooperative OFDMA hexagon cell, in which the base station is in the centre and $K$ relays are equally located on a circle which has the same centre with the cell. Besides, the radius of this circle $r_{2}$ is smaller than the hexagon cell radius $r_{1}$. A total number of $M$ users are deployed uniformly in the cell, $M_{d}$ of which connect directly to the base station while the rest $M_{r}$ ones communicate through a certain relay, $M_{d}, M_{r}=0,1, \ldots, M$. In other words, users can communicate either in a non-cooperative or in a cooperative mode. The total bandwidth of the system is $B$, which is equally divided into $N$ orthogonal subcarrier. The channel is frequency-selective Rayleigh fading. Around this cell there are $L$ co-channel cells. The distribution of relays and users of all cells is similar. Figure 1 shows the cell layout.

We assume all channel state information (CSI) can be perfectly be known by the base station, relays and users. Thus, $\forall k \in\{1,2, \ldots, K\}, m \in\{1,2, \ldots, M\}, n \in\{1,2, \ldots, N\}$, for the $n$th subcarrier, the channel coefficients between base station and the $m$ th user, the $k$ th relay and the $m$ th user, the base station and the $k$ th relay are denoted by $h_{d, m}^{n}$, $h_{a, k m}^{n}$ and $h_{b, k}^{n}$, respectively. The notation $a$ means the first time slot, while the notation $b$ denotes the second one. Meanwhile, the notation $d$ corresponds with the users which are transmitting directly with the base station. The noise variance in the channel is $\sigma^{2}$.

In this article, the relays work under DF mode. In the odd time slot, a cooperative user transmits information to its corresponding relay. And in the next even slot, the relay decodes the signal received in the odd slot and forwards it to the base station. For the $n$th subcarrier, the transmitting power between the $m$ th user and the $k$ th relay in the odd slot can be denoted as $p_{k m}^{n}(1)$, and the transmitting power between the $k$ th relay and the base station in the next even slot is $p_{k m}^{n}(2)$.

Therefore, for the cooperative users, in DF mode, the instantaneous rate of relay-user $(k, m)$ on the $n$th subcarrier can be denoted as

$$
c_{k m}^{n}=\frac{B}{2 N} \min \left\{\log _{2}\left(1+\frac{p_{k m}^{n}(1)\left|h_{a, k m}^{n}\right|^{2}}{\sigma^{2}+I_{a}}\right), \log _{2}\left(1+\frac{p_{k m}^{n}(2)\left|h_{b, k}^{n}\right|^{2}}{\sigma^{2}+I_{b}}\right)\right\}
$$




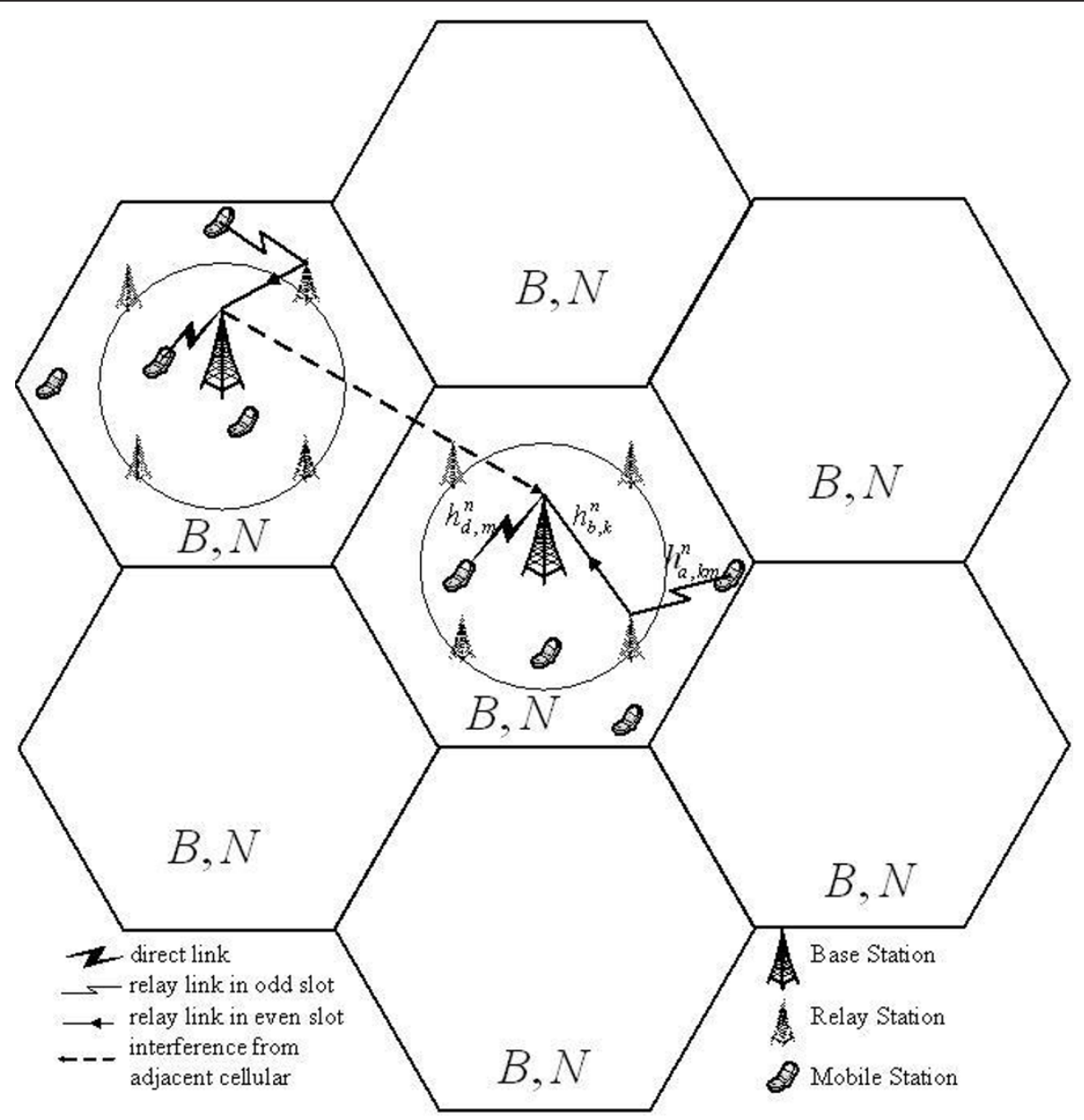

Figure 1 Demonstration of the system structure and the working process of the multi-cell uplink OFDMA system.

where $\quad I_{a}=\sum_{i \in L} \operatorname{pow}_{k m}^{n}(1, l)\left|h h_{a, k m}^{n}(l)\right|^{2} \quad$ and $I_{b}=\sum_{i \in L} \operatorname{pow}_{k}^{n}(2, l)\left|h h_{b, k}^{n}(l)\right|^{2}$ denote the co-channel interference from the $l$ th adjacent cell cooperative users in the odd and even time slots, respectively, $\forall k \in\{1,2, \ldots, K\}$. For the $n$th subcarrier, $h h_{a, k m}^{n}(l)$ is the channel coefficient between the $k$ th relay and the $m$ th user in the $l$ th adjacent cell, and $h h_{b, k}^{n}(l)$ is the channel coefficient between the base station and the $k$ th relay, in addition of $\operatorname{pow}_{k m}^{n}(i, l)$ which denotes the transmit power from the $l$ th adjacent cell in the $i$ th slot, $i=1,2$.

On the other hand, by denoting $p_{0 m}^{n}(1)$ and $p_{0 m}^{n}(2)$ as the transmitting power of non-cooperative users in the odd and even time slots, respectively, we can extend the direct users' instantaneous rate.

$$
c_{0 m}^{n}=\frac{B}{2 N}\left[\log _{2}\left(1+\frac{p_{0 m}^{n}(1)\left|h_{d, m}^{n}\right|^{2}}{\sigma^{2}+I_{0}}\right)+\log _{2}\left(1+\frac{p_{0 m}^{n}(2)\left|h_{d, m}^{n}\right|^{2}}{\sigma^{2}+I_{0}}\right)\right]
$$

where $I_{0}=\sum_{i \in L} \operatorname{pow}_{0 m}^{n}(i, l)\left|h h_{d, m}^{n}(l)\right|^{2}$ denotes the cochannel interference from the $l$ th adjacent cell noncooperative users. Similarly, the transmitting power of non-cooperative user in the $i$ th slot can be denoted as $\operatorname{pow}_{0 m}^{n}(i, l)$, and $h h_{d, m}^{n}(l)$ is the channel coefficient between the base station and the $m$ th user, $i=1,2$.

\section{Solution on relay selection, subcarrier and power allocation}

As is known to us, subcarrier and power allocation impose great influence on system performance. In uplink cooperative OFDMA system, subcarrier is public resource which is in competition among different users. However, power of each user can be controlled independently according to their own demand and power capacity. So, we develop a semi-distributed allocation framework, in which we are pursuing for the combination of merits of centralized and distributed schemes, and can effectively achieve the subcarrier and power 
allocation. In the proposed scheme, relay selection can be achieved with subcarrier allocation under the control of a centre, i.e. the base station, while the next step is achieved by each user.

On the other hand, resource allocation, which contains relay selection, subcarrier and power allocation, is an NP-hard problem which has great complexity. For simplicity, the division of the original problem into two progressive sub-optimal ones, which is at the cost of a loss of optimality, and is convenient for us to solve the problem.

\subsection{Centralized joint relay selection and subcarrier allocation}

The relay selection and power allocation framework can be represented by binary assignment variables $x_{k m}^{n}$, which can alternatively equals to 0 or 1 . While $x_{k m}^{n}=1$, the nth subcarrier is matched for the communications between the mth user and the kth relay, vice versa. If $k=0$, the value of $x_{k m}^{n}$ demonstrates whether the $n$th subcarrier is matched directly for the communications between the $m$ th user and the base station. The $(K+1) \times M \times N$ dimension 0-1 matrix $X=\left(x_{k m}^{n}\right)$ can be defined as the relay-user-subcarrier matching matrix, i.e. the relay selection and subcarrier allocation matrix.

Therefore, we can optimize the framework aiming at maximizing the system capacity. However, water-filling algorithm cannot ensure the QoS of farther users whose CSI is not as good as the nearer ones, i.e. the near-far effect is more obvious [4]. Hence, we can introduce QoS requirement to ensure that each user can reach a minimum rate. The optimization problem for joint relay selection and subcarrier allocation in cooperative OFDMA networks can be formulated as following [2].

$$
\begin{aligned}
& \underset{x_{k m}^{n}}{\arg \max } C_{\text {sum }}=\sum_{k=0}^{K} \sum_{m=1}^{M} \sum_{n=1}^{N} c_{k m}^{n} x_{k m}^{n} \\
& \text { s.t. } \sum_{k=0}^{K} \sum_{m=1}^{M} x_{k m}^{n} \leq 1 \\
& x_{k m}^{n}=\{0,1\} \\
& \sum_{k=0}^{K} \sum_{m=1}^{M} c_{k m}^{n} \geq \bar{c}_{\min }
\end{aligned}
$$

where the $1 \times m$ vector $\bar{c}_{\min }$ denotes the minimum rate of each user.

The optimizing framework is therefore formulated as an integer programming problem. By introducing a $1 \times$ $m$ dual vector $\lambda_{m}$ and introducing sub-gradient method, we can make use of the iterative algorithm proposed in [2] with regardless of power allocation as following.

$$
\begin{aligned}
& x_{k m}^{n}(\text { iter })=\left\{\begin{array}{l}
1,(k, m)=\left(k^{*}, m^{*}\right)=\underset{k, m}{\arg \max }\left[1+\lambda_{m}(\text { iter })\right] c_{k m}^{n} \\
0, \text { o.w. }
\end{array}\right. \\
& \lambda_{m}(\text { iter }+1)=\left[\lambda_{m}(\text { iter })+\alpha(\text { iter })\left(\bar{c}_{\min }-c_{k m}^{n} x_{k m}^{n}(\text { iter })\right)\right]^{+}
\end{aligned}
$$

where iter represents the iterative times, and $\alpha($ iter $)$ means a proper iterative step length which is related to the iterative times.

Theorem 1 [12] If $\sum_{\text {iter }} \alpha$ (iter $) \rightarrow \infty$, and $\alpha($ iter $) \rightarrow 0$ as iter $\rightarrow \infty$, then the optimizing goal can converge upon the optimal value.

According to Theorem 1, we can entitle a non-convergent infinite series to $\alpha($ iter $)$, whose items incline towards zero while the iterative time goes to infinite. Therefore, we can evaluate $\alpha$ (iter) $=\frac{1}{i \text { ter }}$. By iteration of Equations 4 and 5, the optimal solution can be obtained accordingly. It is achieved by the base station in the centre.

\subsection{Distributed power allocation scheme}

When subcarriers' allocation has been finished, we can focus on power allocation with the goal of maximizing each user's rate. We can pay more attention to the even time slot, in which we can distinguish cooperative and non-cooperative modes. By introducing game theory, the power allocation scenario can be corresponded with the three basic components of game theory as following.

Non-cooperative players: the $M_{d}$ non-cooperative users and the $K$ relays which conflict with each other shown in Equations 1 and 2.

The action profile: the transmitting strategy of each player.

The set of utility functions: an income which is related to the rate of each player.

Therefore, the game can be expressed as

$$
\begin{aligned}
& \underset{x_{k m}^{n}}{\arg \max } u_{s}\left(p_{s}^{n}(2)\right) \\
& \text { s.t. } \sum_{n} p_{s}^{n}(2) \leq p_{\max }, \quad s=\left\{1,2, \ldots, K, K+1, \ldots, K+M_{d}\right\}
\end{aligned}
$$

where $p_{s}^{n}(2)$ is the transmitting power of node $s$ in the even time slot, and the elements of $p_{\max }$ are the peak values of the $K$ relays and the $M_{d}$ non-cooperative users.

The design of the utility function exerts great influence on the system performance. For non-cooperative users, with the purpose of adjusting the transmitting power in the even time slot, we can design the utility function of the $m$ th non-cooperative user as 


$$
\begin{aligned}
& u_{m}\left(p_{0 m}^{n}(2)\right)=\frac{B}{N} \sum_{n \in \psi_{d, m}} \log _{2}\left(1+\operatorname{SINR}_{d, m}^{n}(2)\right)-\alpha_{d} \sum_{n \in \psi_{d, m}}\left|h_{d, m}^{n}\right|^{2} p_{0 m}^{n}(2) \\
& \text { s.t. } \sum_{n \in \psi_{d, m}} p_{0 m}^{n}(2) \leq p_{m, \max }
\end{aligned}
$$

where $\psi_{d, m}$ is the subcarrier set of the $m$ th non-cooperative user obtained in Section 3.1, $\operatorname{SINR}_{d, m}^{n}(2)=\frac{p_{0 m}^{n}(2)\left|h_{d, m}^{n}\right|^{2}}{\sigma^{2}+I_{0}}$ is the signal-to-noise-andinterference-ratio (SINR) of the $m$ th non-cooperative user upon the $n$th subcarrier in the even time slot, $\alpha_{d}$ is a non-negative cost factor whose unit is bps/W, in which the notation $\mathrm{W}$ denotes the power unit Watt, and $p_{m \text {, max }}$ denotes the power constraint of the $m$ th noncooperative user. In the utility function above, the first term represents the goal to maximize the $m$ th noncooperative users' rate, and the second term is the cost function which aims at reducing the transmitting power. Particularly, path attenuation is an important factor to determine the cost originating from power consumption. In light of this, the 2-norm of channel coefficients is introduced into the cost function to regulate the power consumption in different channel conditions.

Similarly, for relays which are applied to forward cooperative users' information in the odd slot, the utility function can be designed as following.

$$
\begin{aligned}
& u_{k}\left(p_{k m}^{n}(2)\right)=\frac{B}{N} \sum_{n \in \psi_{, k}} \log _{2}\left(1+\operatorname{SINR}_{k, m}^{n}(2)\right)-\left.\alpha_{r} \sum_{n \in \psi_{r, k}} p_{k m}^{n}(2)||_{b, k}^{n}\right|^{2}-\beta \sum_{n \in \psi_{, k}}\left(\frac{p_{k m}^{n}(2)}{p_{k m}^{n}(1)}-1\right)^{2} \\
& \text { s.t. } \sum_{n \in \psi_{r, k}} p_{k m}^{n}(2) \leq p_{k, \text { max }}
\end{aligned}
$$

where $\psi_{r, k}$ is the subcarrier set of the $k$ th relay for the communication between the $k$ th relay and its corresponding cooperative users, $\operatorname{SINR}_{d, m}^{n}(2)=\frac{p_{k m}^{n}(2)\left|h_{b, k}^{n}\right|^{2}}{\sigma^{2}+I_{b}}$ is the SINR of the $m$ th direct user upon the $n$th subcarrier in the even time slot and $p_{k \text {, max }}$ denotes the power constraint of the $k$ th relay. $\alpha_{r}$ is a non-negative cost factor whose unit is bps/W, while $\beta$ is a non-negative weight factor for the pricing item whose unit is bps. Employing a utility function in the form of (7) will lead to a unilateral SINR increase in even time slot, and the capacity of a relay user is confined by the minimum SINR of the two kinds of time slots. To get the balance in the two slots, the introduction of the third item is necessary. The squared term in the expression grows fast when the SINR in the even slot increases and exceeds that in the odd slot. Thus, the price rises to punish the relay from allocating too much power on that subcarrier [9].

\subsection{The existence of $\mathrm{NE}$}

When it comes to a game model, NE is always our expectation. NE is an important concept in a non-cooperative game, in which each actor is content to keep the present strategy rather than change it. In other words, NE is the profile of each actor's optimal strategy.

Theorem 2 [10] The function must be quasi-concave (or quasi-convex) if it is concave (or convex).

Theorem 3 [10] There is an NE at least in game $G=$ $\left[N,\left\{\mathbf{P}_{i}\right\}, u_{i}(\cdot)\right]$, if

(1) $\mathbf{P}_{i}$ are the sub-sets of $\mathbf{R}^{\mathrm{n}}$ and are non-empty, compact and convex;

(2) $u_{i}$ are continuous in $\mathbf{P}_{i}$;

(3) $u_{i}$ are quasi-concave in $\mathbf{P}_{i}$.

where $N$ is the set of players, $\mathbf{P}_{i}$ and $u_{i}$ are the action profile and the utility function of player $i, i=1,2, \ldots, N$.

According to these theorems, we can give the proof of the existence of NE in the game scenario in this article as follow.

Proof: It is obviously that the first and the second conditions are satisfied according to the expressions of $\mathbf{P}_{i}$ and $u_{i}($.$) . We can focus on the rest one.$

In our game model, for the mth non-cooperative user, we can get the first-order and second-order derivative functions of its utility of Equation 7 as

$$
\begin{aligned}
& \frac{\partial u_{m}\left(p_{0 m}^{n}(2)\right)}{\partial p_{0 m}^{n}(2)}=\frac{B}{N \ln 2} \cdot \frac{\operatorname{SINR}_{d, m}^{n}(2)}{1+\operatorname{SINR}_{d, m}^{n}(2)} \cdot \frac{1}{p_{0 m}^{n}(2)}-\alpha_{d}\left|h_{d, m}^{n}\right|^{2} \\
& \frac{\partial u_{m}^{2}\left(p_{0 m}^{n}(2)\right)}{\partial\left(p_{0 m}^{n}(2)\right)^{2}}=-\frac{B}{N \ln 2} \cdot \frac{1}{\left(1+\operatorname{SINR}_{d, m}^{n}(2)\right)^{2}} \cdot\left(\frac{1+\operatorname{SINR}_{d, m}^{n}(2)}{p_{0 m}^{n}(2)}\right)^{2}<0
\end{aligned}
$$

Similarly, for the $k$ th relay, we can get the first-order and second-order derivative functions of its utility of Equation 9 as

$$
\begin{aligned}
& \frac{\partial u_{k}\left(p_{k m}^{n}(2)\right)}{\partial p_{k m}^{n}(2)}=\frac{B}{N \ln 2} \cdot \frac{\operatorname{SINR}_{k, m}^{n}(2)}{1+\operatorname{SIN}_{k, m}^{n}(2)} \cdot \frac{1}{p_{k m}^{n}(2)}-\alpha_{r}\left|h_{b, k}^{n}\right|^{2}-\frac{2 \beta}{\left(p_{k m}^{n}(2)\right)^{2}}\left(p_{k m}^{n}(2)-p_{k m}^{n}(1)\right) \\
& \frac{\partial u_{k}^{2}\left(p_{k m}^{n}(2)\right)}{\partial\left(p_{k m}^{n}(2)\right)^{2}}=-\frac{B}{N \ln 2} \cdot \frac{1}{\left(1+\operatorname{SINR}_{k, m}^{n}(2)\right)^{2}} \cdot\left(\frac{\operatorname{SINR}_{k, m}^{n}(2)}{p_{k m}^{n}(2)}\right)^{2}-\frac{2 \beta}{\left(p_{k m}^{n}(2)\right)^{2}}<0
\end{aligned}
$$

Therefore, for both relays and direct users, their utility functions are quasi-concave in $p_{k m}^{n}(2)$ and $p_{0 m}^{n}(2)$, respectively. Consequently, the NE exists in the proposed framework.

\subsection{The uniqueness of NE}

The uniqueness of NE demonstrates that each node can reach a converging strategy profile. The authors of [11] proposed an approach to prove it. As a matter of fact, the uniqueness can also be obtained from its original meaning. 
Considering the power strategies profile $\bar{P}$ can be obtained by iteration, whether the positive term series $|\bar{P}(t+1)-\bar{P}(t)|$ can reach its convergence determines the uniqueness of NE.

Proof: For the $m$ th non-cooperative user, let Equation 9 be zero, we can get

$$
p_{0 m}^{n}(2)=\frac{B}{\alpha_{d} N\left|h_{d, m}^{n}\right|^{2} \ln 2} \frac{\operatorname{SINR}_{d, m}^{n}(2)}{1+\operatorname{SINR}_{d, m}^{n}(2)}
$$

Therefore, we can get the value of $p_{0 m}^{n}$ (2) by iteration of Equation 13 and $\operatorname{SINR}_{d, m}^{n}(2)=\frac{p_{0 m}^{n}(2)\left|h_{d, m}^{n}\right|^{2}}{\sigma^{2}+I_{0}}$. When it comes to the iterative expression, as the iterative time $t \rightarrow$ $\infty$, we can get the positive term series

$$
\begin{aligned}
& \left|p_{0 m}^{n}(t+1)-p_{0 m}^{n}(t)\right| \\
& =\left|\frac{B}{\alpha_{d}\left|h_{d, m}^{n}\right|^{2} N \ln 2} \frac{\operatorname{SINR}_{d, m}^{n}(2)(t)}{1+\operatorname{SINR}_{d, m}^{n}(2)(t)}-\frac{B}{\alpha_{d}\left|h_{d, m}^{n}\right|^{2} N \operatorname{Nln} 2} \frac{\operatorname{SINR}_{d, m}^{n}(2)(t-1)}{1+\operatorname{SINR}_{d, m}^{n}(2)(t-1)}\right| \\
& =\frac{B}{\alpha_{d}\left|h_{d, m}^{n}\right|^{2} N \ln 2} \cdot \frac{\left|\operatorname{SINR}_{d, m}^{n}(2)(t)-\operatorname{SINR}_{d, m}^{n}(2)(t-1)\right|}{\left(1+\operatorname{SINR}_{d, m}^{n}(2)(t)\right)\left(1+\operatorname{SINR}_{d, m}^{n}(2)(t-1)\right)}
\end{aligned}
$$

Let $\quad \delta(t)=\frac{1}{\left(1+\operatorname{SINR}_{d, m}^{n}(2)(t)\right)\left(1+\operatorname{SINR}_{d, m}^{n}(2)(t-1)\right)}, \quad$ the equation above can be expressed as

$$
\begin{aligned}
& \left|p_{0 m}^{n}(t+1)-p_{0 m}^{n}(t)\right| \\
& =\frac{B}{\alpha_{d}\left|h_{d, m}^{n}\right|^{2} N \ln 2} \cdot \delta(t) \cdot\left|\operatorname{SINR}_{d, m}^{n}(2)(t)-\operatorname{SINR}_{d, m}^{n}(2)(t-1)\right| \\
& =\frac{B}{\alpha_{d}\left|h_{d, m}^{n}\right|^{2} N \ln 2} \cdot \delta(t) \cdot\left|\frac{\left|h_{d, m}^{n}\right|^{2} p_{0 m}^{n}(2)(t)}{\sigma^{2}+I_{0}}-\frac{\left|h_{d, m}^{n}\right|^{2} p_{0 m}^{n}(2)(t-1)}{\sigma^{2}+I_{0}}\right| \\
& =\frac{B}{\alpha_{d} N \ln 2} \cdot \frac{\delta(t)}{\sigma^{2}+I_{0}} \cdot\left|p_{0 m}^{n}(2)(t-1)-p_{0 m}^{n}(2)(t-2)\right| \\
& =\left(\frac{B}{\alpha_{d} N \ln 2}\right)^{2} \cdot \frac{\delta(t) \delta(t-2)}{\left(\sigma^{2}+I_{0}\right)^{2}} \cdot\left|p_{0 m}^{n}(2)(t-3)-p_{0 m}^{n}(2)(t-4)\right| \\
& =\left(\frac{B}{\alpha_{d} N \ln 2\left(\sigma^{2}+I_{0}\right)}\right)^{\frac{t-1}{2}} \cdot \prod_{i=3}^{t} \delta(i) \cdot\left|p_{0 m}^{n}(2)(2)-p_{0 m}^{n}(2)(1)\right|
\end{aligned}
$$

$\forall \operatorname{SINR}_{d, m}^{n}(2)(t) \neq 0, \delta(t)<1$. So, the second term of the equation above inclines to zero as $t \rightarrow \infty$. If we adjust the factor $\alpha_{d}$ to an adequate value to ensure $\frac{B}{\alpha_{d} N \ln 2\left(\sigma^{2}+I_{0}\right)}<1, \quad$ i.e. $\quad \alpha_{d}>\frac{B}{N \ln 2\left(\sigma^{2}+I_{0}\right)}$, $\lim _{t \rightarrow \infty}\left(\frac{B}{\alpha_{d} N \ln 2\left(\sigma^{2}+I_{0}\right)}\right)^{\frac{t-1}{2}}=0$.

And $\left|p_{0 m}^{n}(2)(2)-p_{0 m}^{n}(2)(1)\right|$ is a definite value. Thus, the right-hand side of Equation 14 inclines towards zero. So, does it if $\frac{B}{\alpha_{d} N \ln 2\left(\sigma^{2}+I_{0}\right)^{2}}=1$. In a word, while $\alpha_{d} \geq \frac{B}{N \ln 2\left(\sigma^{2}+I_{0}\right)}$, the right-hand side of Equation 14 is inclining to zero as $t \rightarrow \infty$, i.e. the series $p_{0 m}^{n}(t+1)-p_{0 m}^{n}(t)$ is absolutely convergent. It means that by iteration, the solution of NE $p_{0 m}^{n}(2)$ can reach a certain value. NE is therefore unique.

Similarly, when it comes to the $k$ th relay, let Equation 11 be zero, we can get the iterative expression of $p_{k m}^{n}(2)$ as following.

$$
p_{k m}^{n}(2)(t+1)=\frac{b+\sqrt{c+d \frac{\operatorname{SINR}_{k, m}^{n}(2)(t)}{1+\operatorname{SINR}_{k, m}^{n}(2)(t)}}}{2 a}
$$

where $\quad a=\frac{2 \beta}{\left(p_{k m}^{n}(1)\right)^{2}}, \quad b=\frac{2 \beta}{p_{k m}^{n}(1)}-\alpha_{r}\left|h_{b, k}^{n}\right|^{2}$,

$$
\begin{aligned}
& c=\frac{4 \beta^{2}}{\left(p_{k m}^{n}(1)\right)^{2}}-\frac{4 \beta \alpha_{r}\left|h_{b, k}^{n}\right|^{2}}{p_{k m}^{n}(1)}+\alpha_{r}\left|h_{b, k}^{n}\right|^{4} \\
& d=\frac{2 \beta B}{\left(p_{k m}^{n}(1)\right)^{2} N} .
\end{aligned}
$$$$
\text { and }
$$

Therefore,

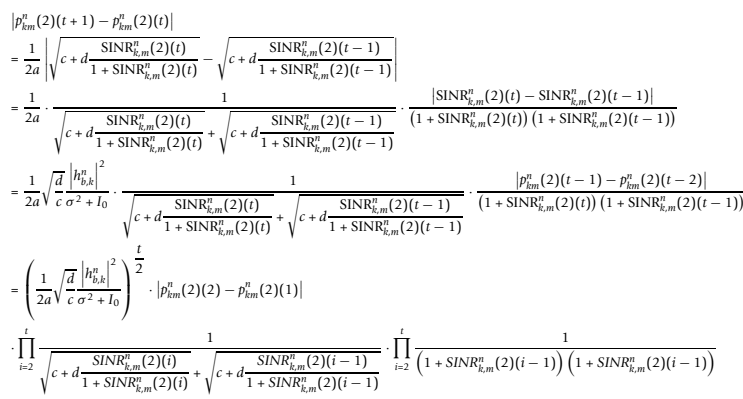

Imitating the explanation of the counterpart of direct users, the second term is a finite value, and the last two terms are smaller than 1 . By adjusting the relationship among $a, c$ and $d$ to make sure $\frac{1}{2 a} \sqrt{\frac{d}{c}} \frac{\left|h_{b, k}^{n}\right|^{2}}{\sigma^{2}+I_{0}} \leq 1$, the series $p_{k m}^{n}(t+1)-p_{k m}^{n}(t)$ is absolutely convergent. NE is therefore unique.

Conclusively, NE of our game approach is unique. In other words, all of the relays and non-cooperative users can automatically choose a strategy of transmitting power, and no one will unilaterally change the power value. But, as far as we are concerned, the Pareto efficiency cannot be ensured because this is a sub-optimal scheme.

\subsection{Relay selection, subcarrier and power allocation algorithm}

According to the discussion above, we can get the progressive optimization of relay selection, subcarrier and power allocation mechanism as Figure 2. 
a. Calculate the CSI and rate according to equation (1) and (2), and initialize the dual vector $\lambda_{m}$ as $\lambda_{m}(0)$.

b. For a given $\lambda_{m}$ (iter), calculate $x_{k m}^{n}$ via equation (4).

c. Update $\lambda_{m}$ (iter) via equation (5).

d. Repeat step $b$ and $c$ until convergence is reached, when $\mid \lambda_{m}($ iter +1$)-\lambda_{m}($ iter $) \mid<\varepsilon . \varepsilon$ is the precision value.
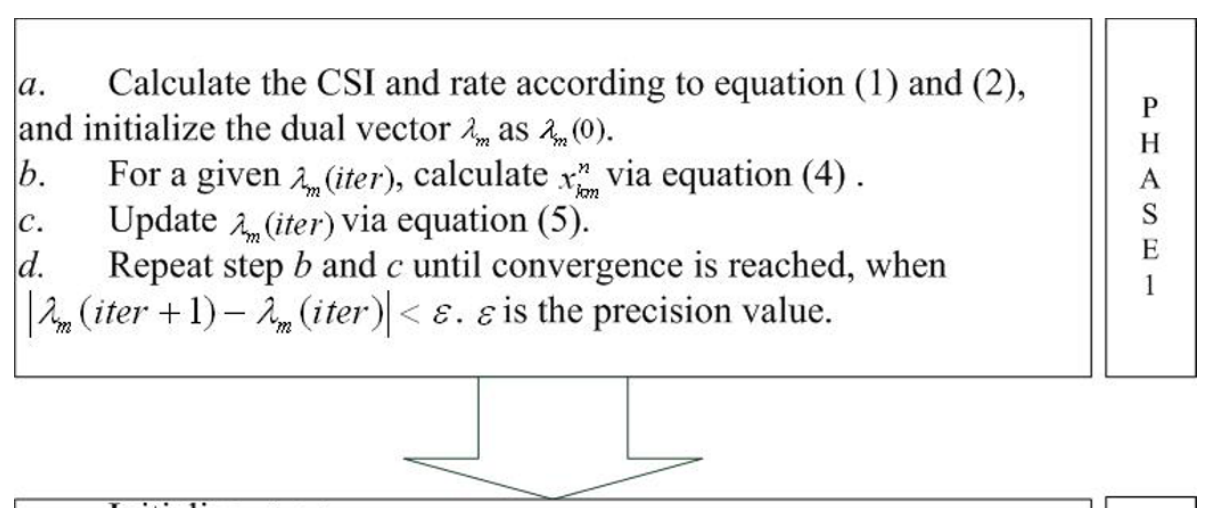

e. Initialize $p_{k m}^{n}(2), k=0,1, \cdots, K$.

f. Obtain the value of $\operatorname{SINR}_{\dot{d}, m}^{n}(2)$ and $\operatorname{SINR} R_{k, m}^{n}(2)$ via $p_{k m m}^{n}(2)$.

g. According to the user-subcarrier matching matrix $X=\left(x_{k n}^{n}\right)$ which is obtained when step $d$ is finished, the direct users and relays update $p_{k m}^{n}(2)$ via equation (13) and (15).

h. Repeat step $f$ and $g$ until convergence is reached, when $\left|p_{k m}^{n}(2)(t+1)-p_{k m}^{n}(2)(t+1)\right|<\eta$. $\eta$ is the precision value..

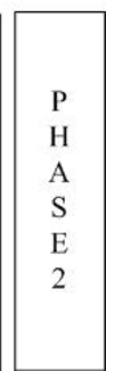

Figure 2 Demonstration of the progressive optimization on relay selection, subcarrier and power allocation. Phase 1 is the joint relay selection and subcarrier allocation based on integer programming, while phase 2 depicts the iterative steps of the game-based power allocation scheme.

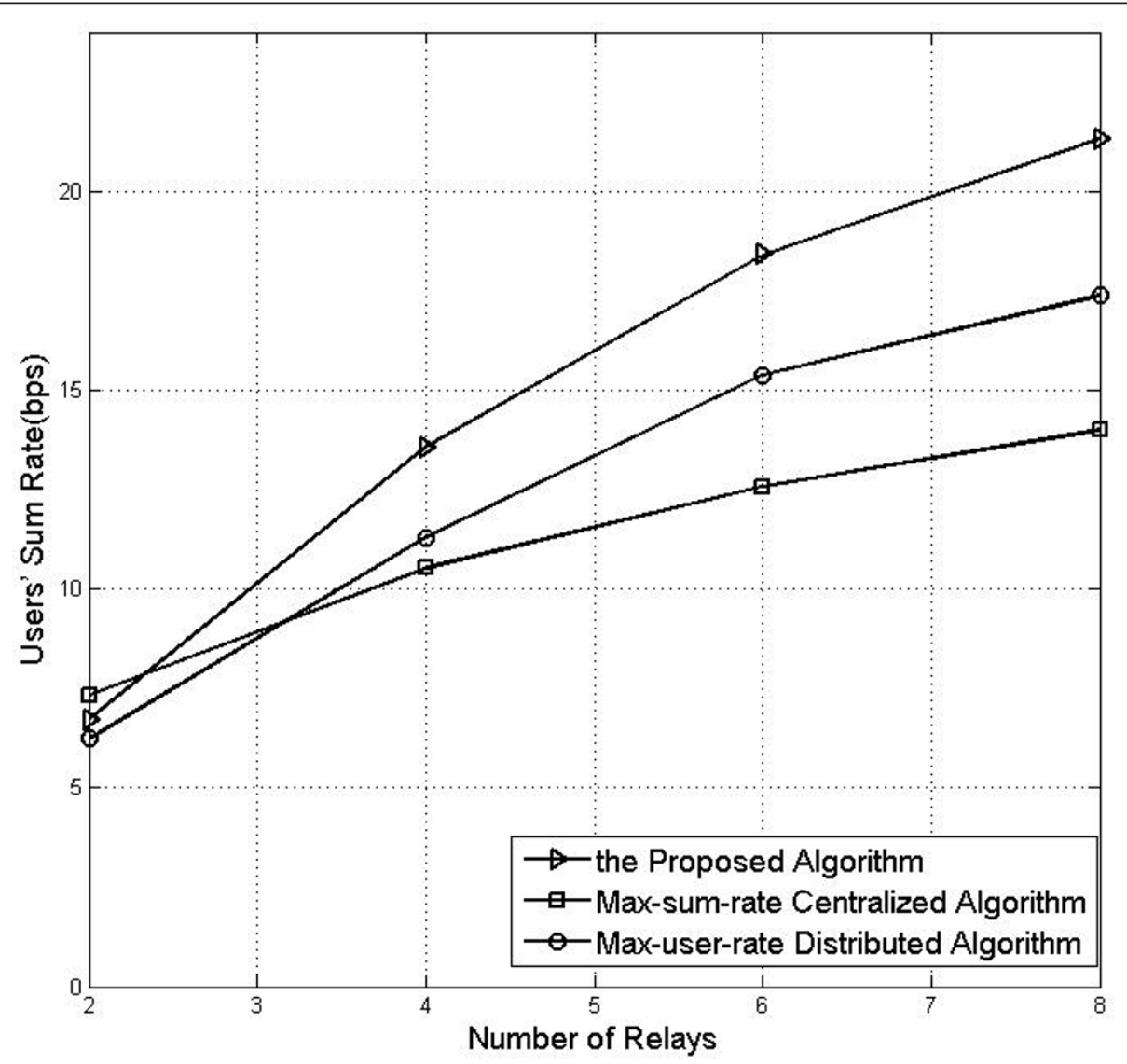

Figure 3 Demonstration of the simulation results of users' sum rate versus number of relays. 


\section{Simulation results}

We consider a cooperative OFDMA cell with $N=64$ orthogonal subcarrier available and a radius of $r_{1}=1 \mathrm{~km}$. DF relays are deployed on a circle with a radius of $r_{2}=$ $0.5 \mathrm{~km}$ at equal angular distance and users are randomly distributed in the cell. The frequency-selective channel consists of six independent paths. Rayleigh multipath adopts Clarke's model. A modified COST231-Hata propagation model is utilized with path loss $128+38 \log (R)$ where $R$ is the distance whose unit is kilometre. The total bandwidth is $1.25 \mathrm{MHz}$ and the power spectral density of noise is $-155 \mathrm{dBm} / \mathrm{Hz}$ [2]. The total power available at all users is $0.05 \mathrm{~W}$, while that at all relays is $0.1 \mathrm{~W} . \alpha_{d}$ and $\alpha_{r}$ are evaluate to 40 and $100 \mathrm{kbps} / \mathrm{W}$, respectively. The power is uniformly allocated on each subcarrier. Six similar cells are allocated around this cell, which have the same subcarriers.

Figure 3 demonstrates the relation of average sum rate versus number of relays under the algorithm in $[2,4]$ and the proposed one, respectively. The total number of the users is 20 . We can see that as the number of relays increases, the sum rate of users increases more obviously under the proposed algorithm. According to our analysis in Section 3.1, on one hand, unilateral pursuit of maximizing system rate under centralized control cannot always meet the requirement of QoS for all distributed users. On the other hand, distributed resource allocation may lead to disorder and low efficiency. For resource which is in competition among users such as subcarrier, it is unfair for the later choosing users to choose subcarriers by turns. Simulation results are consistent with the analysis above. In addition, it demonstrates that the proposed algorithm can efficiently make use of relays to promote the sum rate of users.

Figure 4 discusses the relation of each user's rate versus the distance between base station and users under the algorithm in $[2,4]$ and the proposed one, respectively. The total number of the users is eight with three relays assisting their transmission. As Figure 4 depicts, each user's rate under the proposed algorithm decreases more smoothly than that in the other two algorithms as the distance increases. Therefore, the fairness is well achieved. It is also consistent with the analysis in the paragraph above.

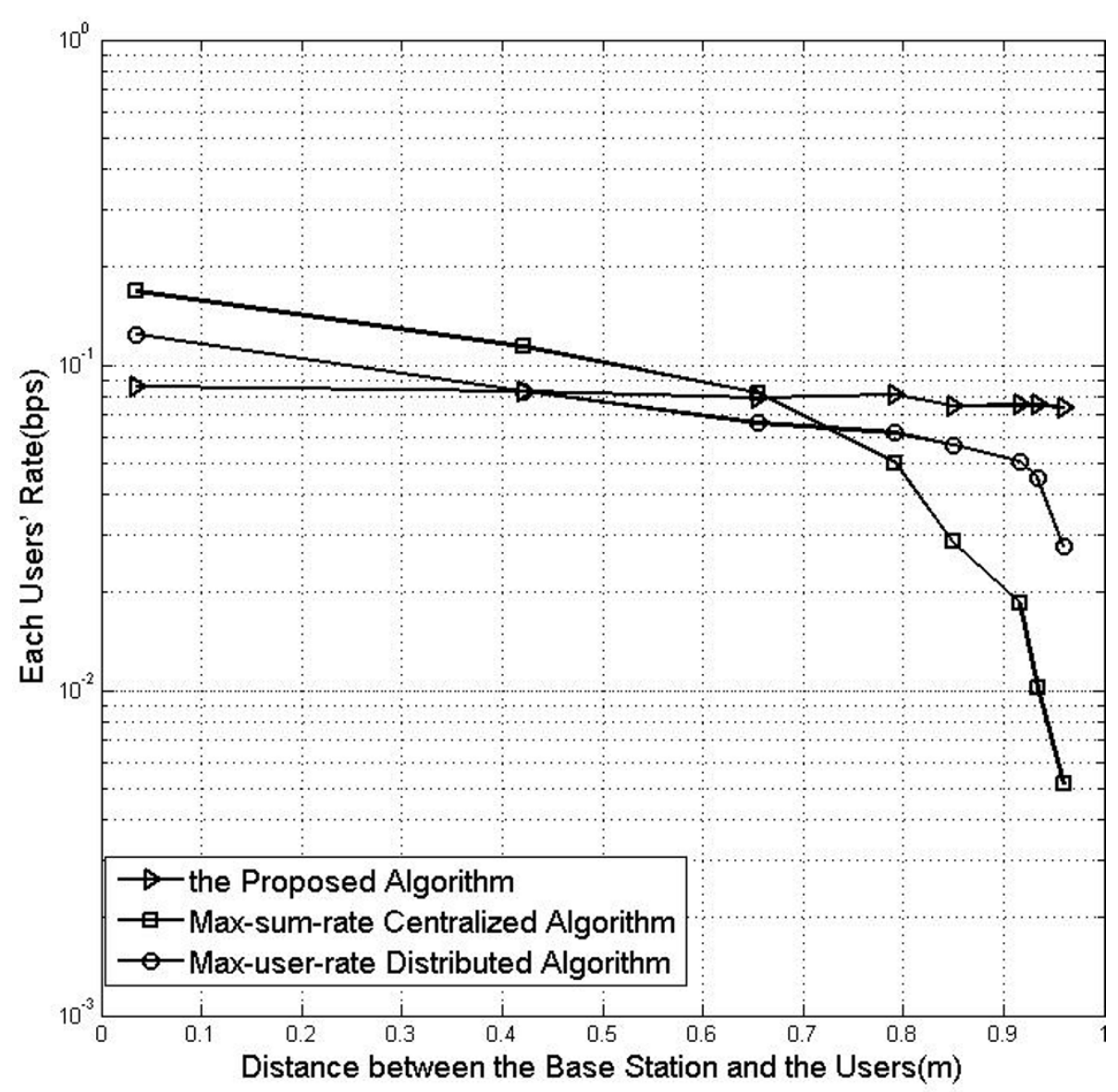

Figure 4 Demonstration of the simulation results of each users' rate versus distance between the base station and the users. 
Figure 5 expresses the relation of average sum rate versus number of relays under the algorithm in $[2,4]$ and the proposed one, respectively. The total number of the relays is 6 . We can see the sum rate of users increases the most sharply under centralized algorithm. It is because that the algorithm in [8] aims at maximizing the system rate, regardless of power consumption of a certain user. The proposed algorithm is a progressive optimal framework, in the second step of which we consider the compromise of user rate maximization and power consumption. Therefore, fairness is achieved at the cost of reduction on the sum rate of users. It also demonstrates that regardless of fairness, centralized framework performs better if the number of user increases.

When it comes to the convergent speed of the proposed scheme, we can discuss this question in the two steps. In the first step, using sub-gradient algorithm, its convergent speed is not so fast [12], totally about 100-300 iterative times. The length of iterative step is related with the convergent speed as analysis in Section 3.2. In the second step, the proposed scheme whose utility function structure similar with [5] can convergence in 10 times.
In a word, the proposed algorithm, which combines the merits of centralized and distributed framework, can efficiently make use of relays to promote the sum rate of users. Meanwhile, fairness improvement is well achieved. Strict mathematic proof of Pareto efficiency is not given, but simulation results demonstrate that the proposed scheme performances promotion on the base of the centralized and distributed schemes.

In addition, when it comes to the feasibility of the proposed algorithm, the CSI should be obtained by the base station in the joint relay selection and subcarrier allocation, and then be conscious of each relay and user. In other words, some overheads are necessary for transmitting the CSI from base station and these distributed nodes. Therefore, the proposed algorithm is suitable for the resource allocation in cooperative OFDMA systems if the cost of channel overhead can be afforded. Otherwise, in energysaving systems, CSI overheads reduce the feasibility of the proposed algorithm.

\section{Conclusion}

In this article, we propose a semi-distributed resource allocation framework for the resource optimization in multi-

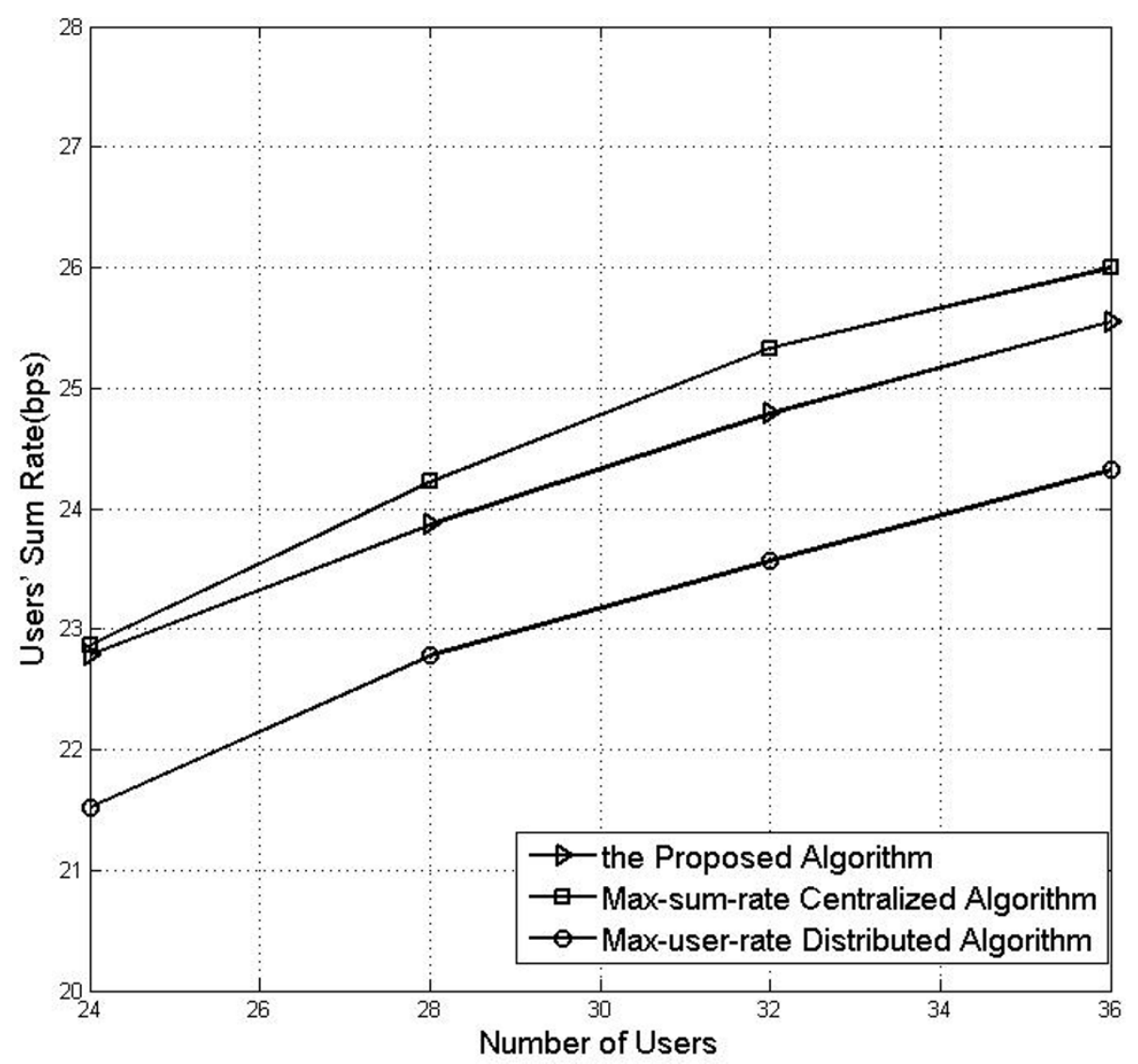

Figure 5 Demonstration of the simulation results of users' sum rate versus number of users. 
cell uplink cooperative OFDM systems in DF mode. Specifically, we model the resource allocation framework as an optimal problem. This optimization problem is divided into two steps. First, using integer programming, we achieve the joint relay selection and subcarrier allocation based on maximizing system sum rate in a centralized way. Second, the distributed power allocation is achieved based on game theory, for cooperative and non-cooperative users, respectively. For cooperative mobile stations, an improved utility is proposed to regulate power allocation in the two time slots. Besides the existence of NE, a new approach for the strict mathematical proof of the uniqueness of NE is proposed. Simulation results demonstrate that the proposed algorithm successfully combines the merits of centralized and distributed framework. It can effectively make use of relays to enhance the sum rate of users as well as achieve the fairness among users.

As a progressive optimization, the semi-distributed scheme combines the merits of centralized and distributed framework in a low complexity. In the future, we are launching on further study about a novel utility function design for system rate elevation, and develop our study towards a transmitting scheme with lower interference, power consumption and CSI overheads. The proof of the Pareto efficiency of transmitting power strategy, as well as the certification of the identity of NE and the optimal solution, needs to be developed as well.

\section{Acknowledgements}

This study was supported by the National Natural Science Foundation of Jiangsu Province (No. BK2010101), the Major National Science \& Technology Specific Project (No. 2010ZX03006-002-04) and the National Natural Science Foundation of China (No. 60972051 and No. 61001107).

\section{Competing interests}

The authors declare that they have no competing interests.

Received: 7 July 2011 Accepted: 15 November 2011

Published: 15 November 2011

\section{References}

1. K Keunyoung, $\mathrm{H}$ Youngnam, $\mathrm{K}$ Seong-Lyun, Joint subcarrier and power allocation in uplink OFDMA systems. IEEE Commun Lett. 6(9), 526-528 (2005)

2. D Zhang, Y Wang, J Lu, QoS aware resource allocation in cooperative OFDMA systems with service differentiation, in IEEE Int Conf Commun., Cape Town South Africa (1 May 2010)

3. Z Han, Z Ji, KJR Liu, Power minimization for multi-cell OFDM networks using distributed non-cooperative game approach, in IEEE Globecom, Texas America. 5, 3742-3747 (November 2004)

4. D Wu, D Yu, Y Cai, Subcarrier and power allocation in uplink OFDMA systems based on game theory, in IEEE Int Conf Neural Netw Signal Process, Wuhan China 522-526 (June 2008)

5. D Yu, D Wu, Y Cai, Subcarrier and power allocation based on game theory in uplink OFDMA systems. J Electron Inf Technol. 4, 775-780 (2010)

6. D Wu, Y Cai, Power allocation in interference relay channels based on noncooperative game theory, in IEEE Int Conf Wireless Commun Signal Process, Nanjing, China (1 November 2009)

7. K-D Lee, T-SP Yum, On Pareto-efficiency between profit and utility in OFDM resource allocation. IEEE Trans Commun. 58(11), 3277-3285 (2010)
8. W Lang, X Yi-sheng, S Egon, Resource allocation in multi-cell OFDM systems based on non-cooperative game, in IEEE Pers Indoor Mobile Radio Commun, Helsinki, Finland (1-5 September 2006)

9. $X Y u, T$ Wu, J Huang, Y Wang, A non-cooperative game approach for distributed power allocation in multi-cell OFDMA-relay networks. IEEE Trans Commun. 43(10), 1920-1924 (2008)

10. AB Mackenzie, LA DaSilva, Game Theory for Wireless Engineers (Morgan \& Claypool Publishers, Washington, 2006)

11. RD Yates, A framework for uplink power control in cellular radio systems. IEEE Sel Areas Commun. 13(7), 1341-1347 (1995). doi:10.1109/49.414651

12. L Wolsey, Integer Programming (Wiley-Interscience Publication, San Francisco, 1998)

doi:10.1186/1687-1499-2011-169

Cite this article as: Hou et al:: Resource allocation based on integer programming and game theory in uplink multi-cell cooperative OFDMA systems. EURASIP Journal on Wireless Communications and Networking 2011 2011:169.

\section{Submit your manuscript to a SpringerOpen ${ }^{\circ}$ journal and benefit from:}

- Convenient online submission

- Rigorous peer review

- Immediate publication on acceptance

- Open access: articles freely available online

- High visibility within the field

Retaining the copyright to your article

Submit your next manuscript at $>$ springeropen.com 